\title{
ADAM33 as a New Biomarker for Invasive Lobular Breast Carcinoma
}

Graciele CM Manica ${ }^{1}$, Edneia A S Ramos ${ }^{1}$, Marco Aurelio Schuler de Oliveira ${ }^{3}$, Fabricio F Costa ${ }^{2}$, Fábio de O Pedrosa ${ }^{3}$, Emanuel M de Souza $^{3}$, Lúcia de Noronha ${ }^{4}$ and Giseli Klassen ${ }^{1 *}$

Department of Basic Pathology, Federal University of Parana, PR, Brazil

${ }^{2}$ Cancer Biology and Epigenomics Program, Children's Hospital of Chicago Research Center and Department of Pediatrics, Northwestern University's Feinberg School of Medicine, Chicago, IL, USA

${ }^{3}$ Department of Biochemistry and Molecular Biology, Federal University of Parana, PR, Brazil

${ }^{4}$ Laboratory of Experimental Pathology, PUC Parana, PR, Brazil

\begin{abstract}
Invasive ductal carcinoma (IDC) and invasive lobular breast carcinoma (ILC) are the most common malignancies compromising the breast tissue in women worldwide. ILCs tend to form metastasisin remote locations, such as the gastrointestinal tract, peritoneal surface and retroperitoneum, compared with IDCs that move preferentially to the lymph nodes, lung, pleura, liver, brain and bones. The histological characteristics of these two tumors are very similar and there is no useful molecular marker to differentiate IDC from ILC to date. In this study, we cloned and expressed ADAM33 recombinant protein cystein rich domain in order to produce polyclonal antibodies and used it as a new immnunohistochemical molecular biomarker that specifically recognizes the ILC breast cancer subtype.
\end{abstract}

Keywords: ADAM33; Breast cancer; Immunohistochemistry; Polyclonal antibody

\section{Introduction}

Invasive ductal (IDC) and Invasive lobular (ILC) breast carcinomas are the most common malignancies of the breast, accounting $80 \%$ and $15 \%$ of all invasive breast tumors, respectively [1,2]. Clinical pathological parameters for aggressiveness such as tumor size, grade and stage are similar for both tumor types $[1,3,4]$. However, clinical follow-up data and the patterns of metastasis suggest that their development and progression are different with lobular tumors being less responsive to neoadjuvant therapy [1,5-7]. The loss of adhesion may explain why ILCs tend to form discohesive histological patterns, a characteristic of primary tumor and metastasis to more remote locations, such as gastrointestinal tract, peritoneal surface and retroperitoneum, compared with IDCs that move preferentially to lymph nodes, lung, pleura, liver, brain and bones $[1,2,7]$. Loss of expression of E-cadherin, the major adhesion protein to the epithelium of the breast, reflects the histological morphology of ILCs [8-10]. However, not all ILCs show complete loss of expression of E-cadherin, implying that other mutations or RNA expression alterations in genes involved in cell-cell adhesion may occur [9].

ADAM33 belongs to the ADAM family of transmembrane glycoprotein and play diverse roles in cell surface remodeling, ectodomain shedding of growth factors and receptors, and mediation of cell-cell and cell-matrix interactions [11,12]. Seniski et al. [13] showed that the ADAM33 promoter hypermethylation was present in $76.2 \%$ of ILC compared with $25.5 \%$ in IDC $(p=0.0002)$. Based on these results, the ADAM33 gene silencing may be related to the discohesive histological nature of ILCs. Previously, we suggested that the ADAM33 promoter methylation may be useful to differentiate ILCs from IDCs.

In this study, we cloned the ADAM33 cysteine rich domain, expressed and purified the recombinant protein. The recombinant protein was then used to produce polyclonal antibodies in order to validate ADAM33 proteinas a new molecular biomarker for ILCs using immunohistochemistry assays.

\section{Materials and Methods}

\section{Cloning the cystein rich domain of ADAM33}

The PCR amplification of the ADAM33 cysteine rich domain was performed from a cDNA library of normal breast cells using the forward primer 5'ACG GCT ACC TGG TAC CAC C, and the reverse primer 5' GCA GGA AGG CAT TGT GGT TT. The purified ADAM33 PCR product was ligated into the $\mathrm{pGEM}^{\circledR}$ Teasy vector (Promega, Brazil). The ADAM33-pGEM ${ }^{\oplus}$ Teasy construct was transformed into E. coli DH10B by electroporation [14]. One clone was selected and sequenced. This clone, named pGM04, was used for plasmid DNA purification using the QiaexII kit according to the manufacturer's instructions (Qiagen, USA). The purified plasmid was digested with the EcoRI (Invitrogen), and subcloned in a pET28a (Novagen) vector. This construction named pGM05 was confirmed by sequencing in an ABI3100 sequencer (Applied Biosystems).

\section{ADAM33 recombinant expression and purification}

The pGM05 plasmid was transformed into E. coli BL21 Ai (Invitrogen). The transformed E. coli strain was grown in $3 \mathrm{ml}$ of LB medium overnight at $37^{\circ} \mathrm{C}$ shaking at $120 \mathrm{rpm}$. Approximately, 1:1000 dilution of the culture was then grown in $100 \mathrm{ml} \mathrm{LB}$ medium until reaching an absorbance of 0.6 at $600 \mathrm{~nm}$. At this point, the expression was induced with $1.0 \mathrm{mM}$ of isopropyl- $\beta$-D-1-thiogalactopyranoside (IPTG) and $2 \%$ arabinose for 4 hours at $37^{\circ} \mathrm{C}$ with shaking. The induced

*Corresponding author: Giseli Klassen, PhD, Department of Basic Pathology, Federal University of Parana, Politecnic Center s/n, Rua Francisco H. dos Santos s/n, P.O. Box 19046, Postal Code 81531-990, Curitiba, 81531-990, Brazil, Tel: 55 41-33611550/55-41-91539778; E-mail: giseli@ufpr.br

Received September 21, 2012; Accepted November 27 2012; Published November 29, 2012

Citation: Manica GCM, Ramos EAS, de Oliveira MAS, Costa FF, de O Pedrosa F et al. (2013) ADAM33 as a New Biomarker for Invasive Lobular Breast Carcinoma J Cancer Sci Ther 5: 014-017. doi:10.4172/1948-5956.1000178

Copyright: (c) 2013 Manica GCM, et al. This is an open-access article distributed under the terms of the Creative Commons Attribution License, which permits unrestricted use, distribution, and reproduction in any medium, provided the original author and source are credited. 
cells expressing the ADAM33 recombinant proteins were collected by centrifugation, resuspended in $30 \mathrm{ml}$ buffer A ( $50 \mathrm{mM}$ Tris- $\mathrm{HCl}, \mathrm{pH}$ 7.8; $50 \mathrm{mM} \mathrm{NaCl}$; urea $8 \mathrm{M}, 20 \mathrm{mM}$ imidazole), and sonicated in an ice-bath of 6 cycles of 20 seconds. The extracts were then clarified by centrifugation at $30,000 \times \mathrm{g}, 30 \mathrm{~min}$ at $4^{\circ} \mathrm{C}$ and the soluble fraction was loaded onto a $1 \mathrm{ml} \mathrm{Hi-Trap} \mathrm{Ni-Chelating} \mathrm{column} \mathrm{(GE} \mathrm{Healthcare)} \mathrm{and}$ pre-equilibrated with buffer A using an Äkta system (GE Healthcare). The column was washed with $10 \mathrm{~mL}$ of buffer $\mathrm{A}$ and proteins were eluted using $20 \mathrm{ml}$ of an imidazole gradient (from $20 \mathrm{mM}$ to $1 \mathrm{M}$ ).

\section{Polyclonal antibody production}

Four female Balb/c mice (45 days old) were immunized three times with purified human recombinant ADAM33 within a fourteen day interval; subcutaneously, each purified protein injection was administered in a concentration of approximately $100 \mu \mathrm{l}$. The first injection contained $60 \mu \mathrm{g}$ of purified ADAM33 in Freund's complete adjuvant (Sigma-Aldrich). The second and third injections contained $60 \mu \mathrm{g}$ in Freund's incomplete adjuvant (Sigma-Aldrich). The immunization and bleeding protocols were examined and approved by the UFPR Animal Ethics Committee (Process 23075.010136/2010-20). The mice were bled on day 60 , and the sera were assayed by Western blot.

\section{Protein analysis}

Protein electrophoresis was carried out by SDS-PAGE 12\% [15] and gels were stained with Coomassie Blue. Protein concentrations were determined by the Bradford assay using bovine serum albumin as a standard [16].

\section{Reactivity of polyclonal antibodies for ADAM33 protein from breast cancer cell lines}

The specificity of the polyclonal antibodies was confirmed by Western Blot, using the extract of protein samples of breast cancer cell lines. The breast cancer cell lines were all obtained from the Ludwig Institute for Cancer Research (São Paulo, Brazil) and were cultured in RPMI 1640 medium containing $10 \%$ fetal bovine serum at $37^{\circ} \mathrm{C}$ in a humidified incubator with 5\% $\mathrm{CO}_{2}$. The cell lines MCF7 and PMC42 have positive ADAM33 expression and MDA-MB-231 was negative for this gene [13], and therefore, was used as positive and negative controls to test the polyclonal antibody. Breast cancer cell line protein lysates were obtained from $1.2 \times 107$ cells in lysis buffer $(50 \mathrm{mM}$ Tris- $\mathrm{HCl}$, $\mathrm{pH} 7.4,0.5 \%$ Triton X-100 and $0.2 \%$ sodium deoxycholate) containing protease inhibitors (Kit Halt TM Thermo Scientific) and $1 \mu$ EDTA 0.5 M. The cell pellets were subjected to sonication and protein samples were used in the Western Blot protocol [14]. Polyclonal antibodies of ADAM33 were used in a 1:10.000 dilution. The horseradish peroxidase (HRP) conjugated light chain-specific anti-mouse IgG secondary antibody (GE Healthcare) was used in concert with a chemiluminescent substrate (GE Healthcare) for signal detection.

\section{Immunohistochemistry}

Immunohistochemistry (IHC) reactions were performed in sections of breast cancer from a tissue microarray (TMA) block [17]. The TMA consisted of 49 breast cancer cases, 38 IDC samples and 11 ILC samples. The primary antibody used was the serum from mice immunized with the recombinant ADAM33 cysteine rich domain diluted at 1:250; and the secondary antibody was the advance link (Dako ๑). The counter-coloration was carried out with Harris hematoxylin for 5 minutes. The visualization of the samples was performed in BX50 light microscope (Olympus ${ }^{\star}$ ) with a camera (DinoLyfe ${ }^{\star}$ ). The software used to obtain digital images was the Image Pro Plus 4.5. Each section was scanned with an objective of $400 \mathrm{x}$ magnification. In the negative control no ADAM33 polyclonal antibody was added to the reaction.

\section{Statistical analyses}

The statistical analyses were performed using a chi-squared test and Fisher's exact test. Statistical significance was assumed for a $\mathrm{p}<0.05$.

\section{Results}

The ADAM33 cysteine rich domain PCR product was cloned into the $\mathrm{pGEM}^{\circledR}$ Teasy and the EcoRI fragment from the pGM04 plasmid were used for subcloning in the pET28a plasmid (data not shown).

E. coli BL21 Ai strain was transformed with a plasmid named pGM05 allowed to purify about $60 \mathrm{mg}$ of recombinant ADAM33 cysteine rich domain (Figure 1).

The specificity of the mouse polyclonal antibodies against human ADAM33 protein was confirmed by Western blot, using protein samples of breast cancer cell lines. The results showed that mouse sera have high specificity for ADAM33 recombinant protein since no signal was detected in the MDA-MB-231 cell line, which does not express the ADAM33 gene [13] (Figure 2). Moreover, MCF7 and PMC42 cell lines that express the ADAM33 gene showed a single and strong signal of approximately $37 \mathrm{kDa}$ for this protein. All samples tested were positive for $\beta$-actin protein that was used as a positive control (Figure 2).

In order to analyze the specificity of the Anti-ADAM33 polyclonal serum in tissue, immunohistochemistry assay was done using a tissue microarray of breast tumors (TMA), comprising of 38 ductal and 11 lobular tumors. A representative photomicrograph of tumor samples is shown in figure 3. All IDC samples were ADAM33 positive (representative sample was shown in the figure 3B), showing infiltration by invasive ductal carcinoma cells composed of neoplastic ducts, cells with marked atypia, hyperchromatic nuclei and coarse chromatin, ample cytoplasm and indistinct boundaries. Compared with the negative control (Figure 3A), a cytoplasmic and weak membrane signal positivity for anti-ADAM33 was observed in the cancer cells, as evidenced by the brown colour of these structures.

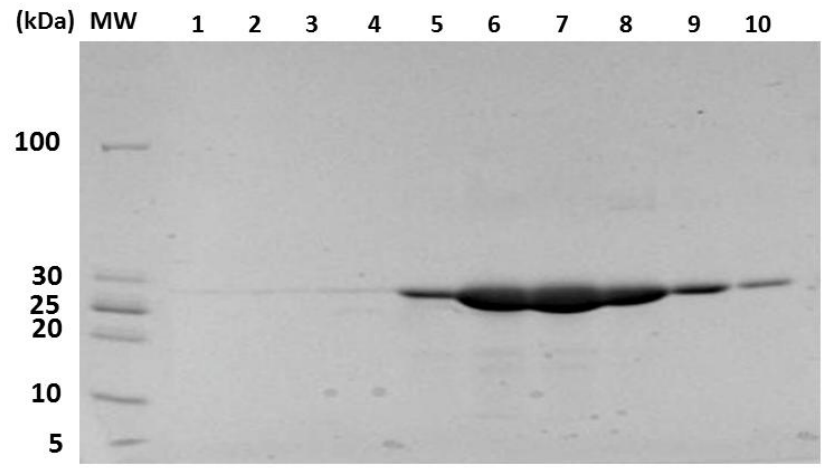

Figure 1: SDS-PAGE gel showing the purification of ADAM33-HisTag. Soluble extract of ADAM33 recombinant protein expressed in E. coli. BL21Ai was purified in a Hi-Trap Ni-Chelating column (GE Healthcare). Some proteins cannot be detected in the flow through fraction (lane 1-4). Recombinant protein was eluted using an imidazole gradient (from $20 \mathrm{mM}$ to $1 \mathrm{M}$ ) (lanes 5-10). Samples were separated by SDS-PAGE and stained with Coomassie blue. 
On the other hand, 7 out of 11 lobular tumors were ADAM33 negative. The cancer cells in the figures $3 \mathrm{C}$ and $3 \mathrm{D}$ show atypical neoplastic cells arranged in solid aggregates, or in a single row and little desmoplastic matrix interspersed in the neoplastic lobules. Figure $3 \mathrm{D}$ shows an example of lobular carcinoma with poor response to polyclonal anti-ADAM33 antibody. It is important to note that there are positive stained cells in the cytoplasm and membrane corresponding to inflammatory cells inside the tumor.

The TMA consisted of 49 cases, with ages ranging from 27 to 84 years. The stages of the breast cancer were I, II or III. Tumor types of 38 IDC (78\%) and 11 ILC (22\%) were used in the study. The

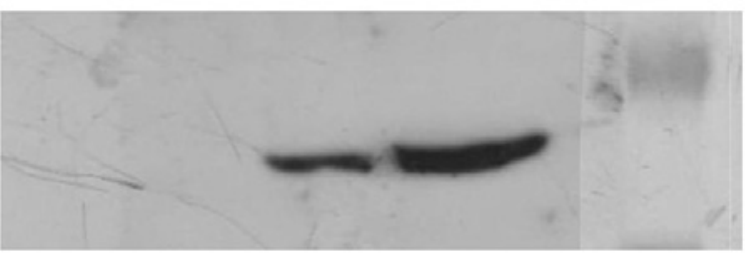

$50 \mathrm{kDa}$

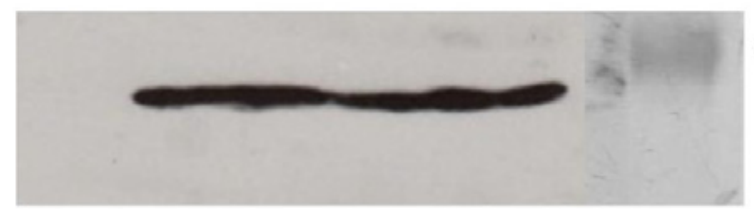

$50 \mathrm{kDa}$

Figure 2: Immunodetection of ADAM33 (top) and constitutive $\beta$-Actin (below) in breast tumor cell lines by Western blot. 1) MDA-MB-231 2) MCF7 3) PMC42. MW corresponding the Molecular weight marker. This result is the representative of four experiments.

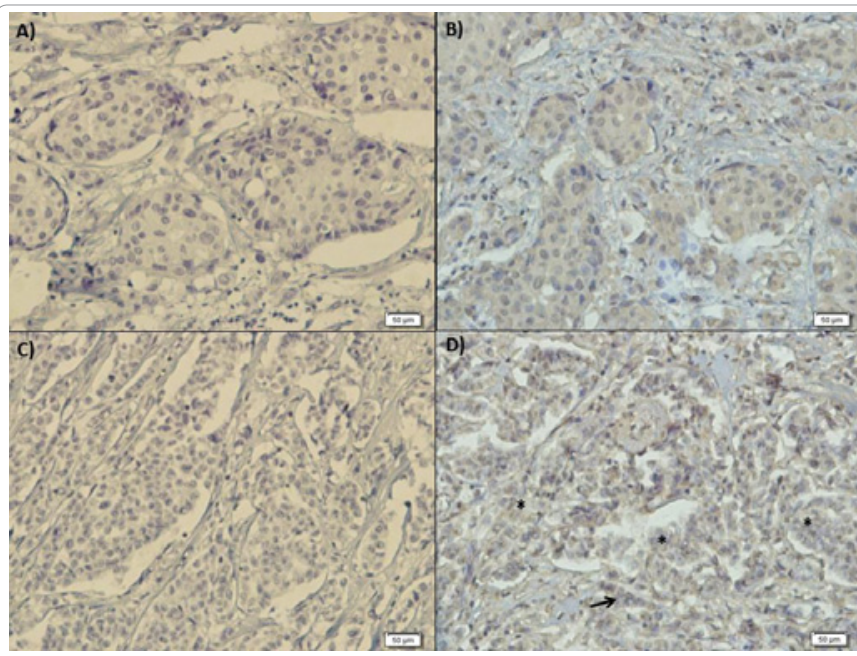

Figure 3: Immunohistochemical assay with the polyclonal antibody antiADAM33. Ductal samples were composed of cells with marked atypia, hyperchromatic nuclei and coarse chromatin, ample cytoplasm and indistinct boundaries. A) IDC negative control, B) IDC anti-ADAM33: cytoplasmic and weak membrane signal positivity for antibody evidenced by the brown color of these structures. For lobular samples we observed atypical neoplastic cells arranged in solid aggregates or in a single row, and a little desmoplastic matrix, interspersed in the neoplastic lobules. C) ILC negative control, D) ILC anti-ADAM33: poor response to polyclonal anti-ADAM33 antibody showing the region with invasive lobular cancer cells in a single row (arrow), ${ }^{*}$ Invasive lobular cancer cells in aggregates, $200 \mathrm{X}$ analysis of the ADAM33 gene hypermethylation was done previously by our group, using MSP analysis [13] and used here to correlate the molecular silencing to the presence or absence of ADAM33 protein staining (Table 1). All the IDC samples were ADAM33 protein positive in IHC. However, 11 out of 38 tumoral samples (Table 1) showed to be hypermethylated in the ADAM33 promoter as seen in our previous work [13]. This result could be explained by other epigenetic mechanisms involved in the silencing of this gene as discussed below.

Nevertheless, the ILC samples were almost negative since 7 samples were totally negative and 4 samples showed a very weak signal for ADAM33 IHC. The ADAM33 promoter of 7 out of 11 ILCs samples were hypermethylated and no ADAM33 protein signal was visualized and this is a statistically significant result ( $\mathrm{p}=0.0004$, Table 1$)$. Finally, the analysis of histological tumor types and the status of ADAM33 protein in IHC were statistically significant $(\mathrm{p}=0.0001)$.

\section{Discussion}

ILCs is the second most common type of breast cancer, accounting for $10-15 \%$ of breast cancers $[1,5,10]$. The immunohistochemistry is an essential tool used in the diagnosis and prognosis of cancers, especially breast tumors. However, there is no biomarker for differentiation of lobular and ductal carcinomas of the breast. In filtrating lobular carcinoma (ILC) and infiltrating ductal carcinoma (IDC) are similar in many aspects and their histologic features occasionally overlap [5]. Despite the similarities, some clinical follow-up data and the patterns of metastasis suggest that ILC and IDC are biologically distinct [1]. Most breast cancer research has focused almost exclusively in the ductal sub type differences or has not stressed the biologic or distinctions between breast carcinoma subtypes $[5,18]$.

In this study, we aimed to test if the ADAM33 protein could be used as a molecular marker for ILCs since its expression is epigenetically down regulated specifically in this breast cancer type [13]. In order to accomplish this, we initially generated a reliable polyclonal antibody using a truncated form of ADAM33 gene; which encodes for the cysteine rich domain. The recombinant protein was expressed in E. coli BL21 Ai, purified and used to immunize Balb-c mice. The serum specificity for the truncated ADAM33 protein was then challenged against crude extract of the breast cancer cell line MDA-MB-231, which was previously shown to be silenced by DNA hypermethylation [13] and has no signal of the polyclonal anti-ADAM33. Moreover, MCF7 and PMC42 cell lines that express the gene and are promoter methylation free, showed a single signal of approximately $37 \mathrm{kDa}$ in a Western blot. These results reinforce that the polyclonal antibodies produced were specific for the ADAM33 protein. The signal generated from the extract of cancer cell lines expressing the ADAM33 gene was similar to that found in other studies [12]. ADAM33 transcripts can

\begin{tabular}{|c|c|c|c|c|c|c|c|}
\hline \multirow[t]{2}{*}{ Variables } & \multirow[t]{2}{*}{$\mathbf{N}$} & \multicolumn{2}{|c|}{ ADAM33 IHC } & \multirow[t]{2}{*}{$p$} & \multicolumn{2}{|c|}{ ADAM33 } & \multirow[t]{2}{*}{$p$} \\
\hline & & Neg & Pos & & U & $\mathbf{M}$ & \\
\hline \multicolumn{8}{|c|}{ ADAM33 IHC } \\
\hline Positive & 39 & - & - & \multirow{2}{*}{-} & 29 & 10 & \multirow{2}{*}{0.0004} \\
\hline Negative & 7 & - & - & & 0 & 7 & \\
\hline \multicolumn{8}{|c|}{ Histologicaltype } \\
\hline IDC & 38 & 0 & 38 & \multirow{2}{*}{$<0.0001$} & 25 & 11 & \multirow{2}{*}{0.0765} \\
\hline ILC & 11 & 7 & 4 & & 4 & 7 & \\
\hline
\end{tabular}

Neg: negative; pos: positive; U: unmethylated; M: methylated

Table 1: Clinicopathological features of the 49 patients with primary breast carcinomas according to ADAM33 gene methylation and immuhistochemical status. 
be alternatively spliced, giving rise to a number of protein isoforms of varying molecular masses $[11,12,19]$.

The immunohistochemistry assay was done using a tissue microarray of breast tumors comprising 38 ductal and 11 lobular tumors. The IDCs samples that were positive to IHC presented a brown colour observed in the cytoplasm and membrane which was absent in the desmoplasic region, indicating that the immunolocalization of the antibody was also specific. The weak signal from the membrane in all the samples may be indicative of the observations made by Garlisi et al. [11] which showed that only $10 \%$ of the protein part produced is directed to the extracellular membrane. The IDCs staining was in agreement with our previous finding, showing that the ADAM33 promoter is mostly unmethylated in IDCs [13]. However, 11 out of 38 samples were positive for the ADAM33 protein and presented promoter hypermethylation (Table 1). This result could be explained by the epigenetic mechanisms, such as gene silencing involved in short DNA methylation and histone tail modifications. Therefore, it is possible that a necessary repressive histone mark was erased in these tumor samples. Another possibility involves a mono allelic hypermethylation not studied yet in this gene promoter region [20]. This result reinforces the importance of the use of this protein as a biomarker more than the molecular signalization obtained from DNA methylation at least for the ADAM33 gene.

The methylation status of the ADAM33 promoter visualized before by us [13] corroborates with the absence of staining of ADAM33 in the ILC tumor samples, since some samples had no promoter methylation $(p=0.0004)$.This result could indicate that other mechanisms not involving DNA methylation are silencing ADAM33 in these tumor samples.

Using the ADAM33 polyclonal antibody in immunohistochemistry made possible to one more time confirm the difference between IDC and ILC, since IDC was always positive for this reaction and ILC was usually weak or completely negative. These results corroborate the hypothesis that the ADAM33 protein could be used as a molecular biomarker for breast cancer and, more specifically, to differentiate both breast cancer types, since these results were statistically significant $(\mathrm{p}=0.0001)$. This is the first study proposing the use of the ADAM33 protein as a molecular marker by immunohistochemistry to differentiate the two main types of breast cancer. In addition, these novel polyclonal anti-ADAM33 antibodies may be useful in further studies to evaluate the function of ADAM33 in normal physiological states, different types of cancer and other diseases.

\section{Acknowledgements}

This work was supported by FundaçãoAraucária (19532/183/10 process), the Brazilian Research Council (CNPq) and CAPES.

Fabricio F. Costa was supported by the Maeve McNicholas Foundation and the Falk Brain Tumor Foundation.

\section{References}

1. Yoder BJ, Wilkinson EJ, Massoll NA (2007) Molecular and morphologic distinctions between infiltrating ductal and lobular carcinoma of the breast. Breast J 13: 172-179.

2. Turashvili G, Bouchal J, Baumforth K, Wei W, Dziechciarkova M, et al. (2007) Novel markers for differentiation of lobular and ductal invasive breast carcinomas by laser microdissection and microarray analysis. BMC Cancer 7: 55.
3. Lopez-Garcia MA, Geyer FC, Lacroix-Triki M, Marchió C, Reis-Filho JS (2010) Breast cancer precursors revisited: molecular features and progression pathways. Histopathology 57: 171-192.

4. Raouf $A$, Sun $Y$, Chatterjee $S$, Basak $P$ (2012) The biology of human breast epithelial progenitors. Semin Cell Dev Biol 23: 606-612.

5. Turashvili G, Bouchal J, Burkadze G, Kolar Z (2005) Mammary gland development and cancer. Cesk Patol 41: 94-101.

6. Eroles P, Bosch A, Pérez-Fidalgo JA, Lluch A (2012) Molecular biology in breast cancer: intrinsic subtypes and signaling pathways. Cancer Treat Rev 38: $698-707$

7. Carcoforo P, Raiji MT, Langan RC, Lanzara S, Portinari M, et al. (2012 Infiltrating Lobular Carcinoma of the Breast Presenting as Gastrointestinal Obstruction: A Mini Review. J Cancer 11-15

8. Nishizaki T, Chew K, Chu L, Isola J, Kallioniemi A, et al. (1997) Genetic alterations in lobular breast cancer by comparative genomic hybridization. Int J Cancer 74: 513-517.

9. Harigopal M, Shin SJ, Murray MP, Tickoo SK, Brogi E, et al. (2005) Aberrant E-cadherin staining patterns in invasive mammary carcinoma. World J Surg Oncol 3: 73

10. Kristiansen G, Pilarsky C (2008) Molecular pathology of Invasive Lobular Breast Carcinoma. Breast Dis 30: 9-14.

11. Garlisi CG, Zou J, Devito KE, Tian F, Zhu FX, et al. (2003) Human ADAM33: protein maturation and localization. Biochem Biophys Res Commun 301: 3543.

12. Powell RM, Wicks J, Holloway JW, Holgate ST, Davies DE (2004) The splicing and fate of ADAM33 transcripts in primary human airways fibroblasts. Am J Respir Cell Mol Biol 31: 13-21.

13. Seniski GG, Camargo AA, lerardi DF, Ramos EA, Grochoski M, et al. (2009) ADAM33 gene silencing by promoter hypermethylation as a molecular marker in breast invasive lobular carcinoma. BMC Cancer 9: 80.

14. Sambrook DW, Russel J (2001) Molecular Cloning: A laboraroty manual.

15. Laemmli UK (1970) Cleavage of structural proteins during the assembling of the head of the bacteriophage T7. Nature.

16. Bradford MM (1976) A rapid and sensitive method for the quantitation of microgram quantities of protein utilizing the principle of protein-dye binding Anal Biochem 72: 248-254.

17. Schuler SL, Gurmini J, Cecílio WA, Viola de Azevedo ML, Olandoski M, et al. (2008) Hepatic and thymic alterations in newborn offspring of malnourished rat dams. JPEN J Parenter Enteral Nutr 32: 184-189.

18. Weigelt B, Geyer FC, Reis-Filho JS (2010) Histological types of breast cancer: how special are they? Mol Oncol 4: 192-208.

19. Haitchi HM, Powell RM, Shaw TJ, Howarth PH, Wilson SJ, et al. (2005) ADAM33 expression in asthmatic airways and human embryonic lungs. Am Respir Crit Care Med 171: 958-965

20. Grønbaek K, Hother C, Jones PA (2007) Epigenetic changes in cancer. APMIS 115: 1039-1059. 